\title{
Effect of growth pressure on coalescence thickness and crystal quality of GaN deposited on $4 \mathrm{H}-\mathrm{SiC}$
}

\author{
Piotr Caban a,b,*, Wlodek Strupinski ${ }^{\mathrm{a}}$, Jan Szmidt ${ }^{\mathrm{b}}$, Marek Wojcik ${ }^{\mathrm{a}}$, Jaroslaw Gaca ${ }^{\text {a }}$, Ozgur Kelekci ${ }^{\mathrm{c}}$, \\ Deniz Caliskan ${ }^{\mathrm{c}}$, Ekmel Ozbay ${ }^{\mathrm{c}}$ \\ ${ }^{a}$ Institute of Electronic Materials Technology, Wolczynska 133, Warsaw 01-919, Poland \\ ${ }^{\mathrm{b}}$ Institute of Microelectronics and Optoelectronics, Warsaw University of Technology, Koszykowa 75, Warsaw 00-662, Poland \\ ' Nanotechnology Research Center, Bilkent University, Bilkent 06800, Ankara, Turkey
}

\section{A R T I C L E I N F O}

Available online 25 September 2010

Keywords:

A1. Defects

A1. High resolution X-ray diffraction

A1. Nucleation

A3. Low pressure metalorganic vapor phase

epitaxy

B1. Nitrides

B3. High electron mobility transistors

\begin{abstract}
A B S T R A C T
The influence of growth pressure on the coalescence thickness and the crystal quality of GaN deposited on $4 \mathrm{H}-\mathrm{SiC}$ by low pressure metalorganic vapor phase epitaxy was studied. It was shown that growth pressure has an impact on the surface roughness of epilayers and their crystal quality. GaN coalescence thicknesses were determined for the investigated growth pressures. The GaN layers were characterized by AFM and HRXRD measurements. HEMT structures were also fabricated and characterized. Among the growth pressures studied, 50, 125 and 200 mbar, 200 mbar was found to be most suitable for GaN/SiC epitaxy.
\end{abstract}

(c) 2010 Elsevier B.V. All rights reserved.

\section{Introduction}

Good quality epilayers are required for any electronic device. In the case of GaN on $\mathrm{SiC}$ manufactured for a GaN-based HEMT structure, a good quality means: low surface roughness, low dislocation density, good crystal quality and high resistance. To fulfill these requirements growth parameters have to be optimized; to this end the growth mechanism, especially at early stages, needs to be investigated.

The GaN deposition mechanism was first described by Hiramatsu et al. [1], and it was also investigated by others [2,3]. The influence of reactor pressure on the morphology of GaN deposited on a sapphire was studied by Wickenden et al. [4]. According to their findings at the beginning of this process small islands are created on the substrate surface, which later expand until they join together making a continuous layer. The process of island fusion is usually called coalescence. Growth before coalescence is described as three-dimensional and the one after its completion as two-dimensional. The process of coalescence could be characterized as well by the duration of the process and the thickness of the layer immediately after fusion. These parameters, which can be used interchangeably, are very important from the point of view of the structural quality of the GaN layer [3].
The objective of this work was to determine the influence of growth pressure on the quality of the GaN epilayer and the process of GaN coalescence.

In order to achieve this goal ex situ surface morphology investigations by means of AFM were carried out on the GaN structures. These structures have been deposited so that growth runs were stopped after 1, 6, 10, 15, 28 and 50 min of the GaN deposition at reactor pressures of 50,125 and 200 mbar.

The defect-selective etching of the GaN surface in molten $\mathrm{NaOH}-\mathrm{KOH}$ eutectic with the addition of $10 \%$ of $\mathrm{MgO}$ powder was used in order to reveal dislocation density on the $50 \mathrm{~min}$ grown GaN epilayer surface. The AFM images of the etched epilayers had been used for estimation of dislocation density.

Taking into account the results of the above described investigations, GaN/Al ${ }_{0.23} \mathrm{GaN} / \mathrm{AlN} / \mathrm{GaN}$-based HEMT structures with $1.7 \mu \mathrm{m}$ thick GaN buffer (grown at 50, 125 or 200 mbar for $50 \mathrm{~min}$ ) were prepared on semi-insulating substrates applying the same growth conditions as those used in previous experiments. These structures were characterized by means of high resolution X-ray diffraction (HRXRD) and AFM. Next sample HEMTs devices were processed and electrically characterized to determine the buffer resistivity, which is expected to depend on its crystal quality.

\section{Experiment}

GaN-based structures were grown on silicon carbide substrates in an AIX 200/4 RF-S metalorganic vapor phase epitaxy low-pressure reactor (LP MOVPE). The source gases used for

\footnotetext{
*Corresponding author at: Institute of Electronic Materials Technology, Wolczynska 133, 01-919 Warsaw, Poland.

E-mail address: piotr.caban@itme.edu.pl (P. Caban).
} 
growth of the AlN wetting layer were trimethylaluminium (TMAl, $0.0087 \mathrm{mmol} / \mathrm{min}$ ) and ammonia $\left(\mathrm{NH}_{3}, 53 \mathrm{mmol} / \mathrm{min}\right)$, whereas trimethylgallium (TMGa, $0.0311 \mathrm{mmol} / \mathrm{min})$ and ammonia $\left(\mathrm{NH}_{3}\right.$, $89 \mathrm{mmol} / \mathrm{min}$ ) were used as a source gas for the growth of the GaN layers during the first minute of the deposition, then the TMGa flow was increased to $0.0886 \mathrm{mmol} / \mathrm{min}$ and was kept this way till the end of the growth process. The same situation occurred with the growth temperature, in the beginning it amounted to $1070{ }^{\circ} \mathrm{C}$ for the first minute of GaN deposition and then it was increased to $1110{ }^{\circ} \mathrm{C}$. The process of increasing the temperature took $5 \mathrm{~min}$. After reaching the required level it was kept constant till the end of the deposition process. The reactor pressure for GaN growth was 50, 125 mbar or 200 mbar for different samples.

At the beginning of the deposition process the $100 \mathrm{~nm}$ thick AlN wetting layer was deposited $[5,6]$ at the temperature of $1070{ }^{\circ} \mathrm{C}$ and reactor pressure of $50 \mathrm{mbar}$. These wetting layer growth conditions were kept identical for all analyzed samples.

In the presented study, ( $\left.\begin{array}{llll}0 & 0 & 0 & 1\end{array}\right)$-oriented N-type or semiinsulating ( $\mathrm{SI}$ ) $4 \mathrm{H}-\mathrm{SiC}$ were used as the substrates for epitaxy. Conductive $\mathrm{SiC}$ was utilized as a substrate during the investigations of GaN coalescence, whereas semi-insulating substrates were employed for the HEMTs epitaxial structures. The epitaxial growths were performed on the $\mathrm{Si}$-face of $\mathrm{SiC}$ substrates. Substrates were cut into $1.5 \mathrm{~cm} \times 1.5 \mathrm{~cm}$ pieces, rinsed with alcohol and deionized water and dried in an $\mathrm{N}^{2}$ flow. All of them were research grade and from the same supplier. They did not undergo special surface preparation. The growth process was controlled by in situ reflectance measurements.

\section{Results and discussion}

In order to investigate comprehensively the influence of reactor pressure on the crystal quality of GaN layers, the experiment was divided into steps wherein the following issues were examined:

a) growth mechanism for samples differing in time of deposition and reactor pressure;

b) surface quality and density of dislocations for samples with the same thickness, deposited at different reactor pressures; and c) crystal quality and electrical properties of HEMT structures.

The main idea of part (a) of the experiment was to investigate the differences in morphology of GaN layer appearing during the growth of $\mathrm{GaN} / \mathrm{AlN} / 4 \mathrm{H}-\mathrm{SiC}$ samples performed at different pressures in the reactor chamber, paying special attention to the coalescence stage of the growth process. For ex situ surface morphology investigations the growth runs were stopped after 1 , $6,10,15$ and $28 \mathrm{~min}$ of the GaN deposition at reactor pressures of 50,125 and 200 mbar. The surface morphology of the epilayers was studied using differential interference contrast (DIC) or Nomarski optical microscopy.

It was assumed that the $100 \mathrm{~nm}$ thick AlN wetting layer surface roughness and crystal quality are reproducible from growth to growth (Fig. 1).

In none of the GaN samples that were grown at 50, 125 and 200 mbar for $1 \mathrm{~min}$, was it possible to differentiate between GaN and AlN layers employing only AFM working in the tapping mode. In order to get around this problem it was decided to use a Scanning Electron Microscope (SEM) with an EsB detector for the determination of the presence of GaN seeds on the AlN surface. The method applied was that of Energy and Angle selective (EsB detection) Back Scattered Electrons (BSE) detection. In this case the principle of image formation is based on the detection of

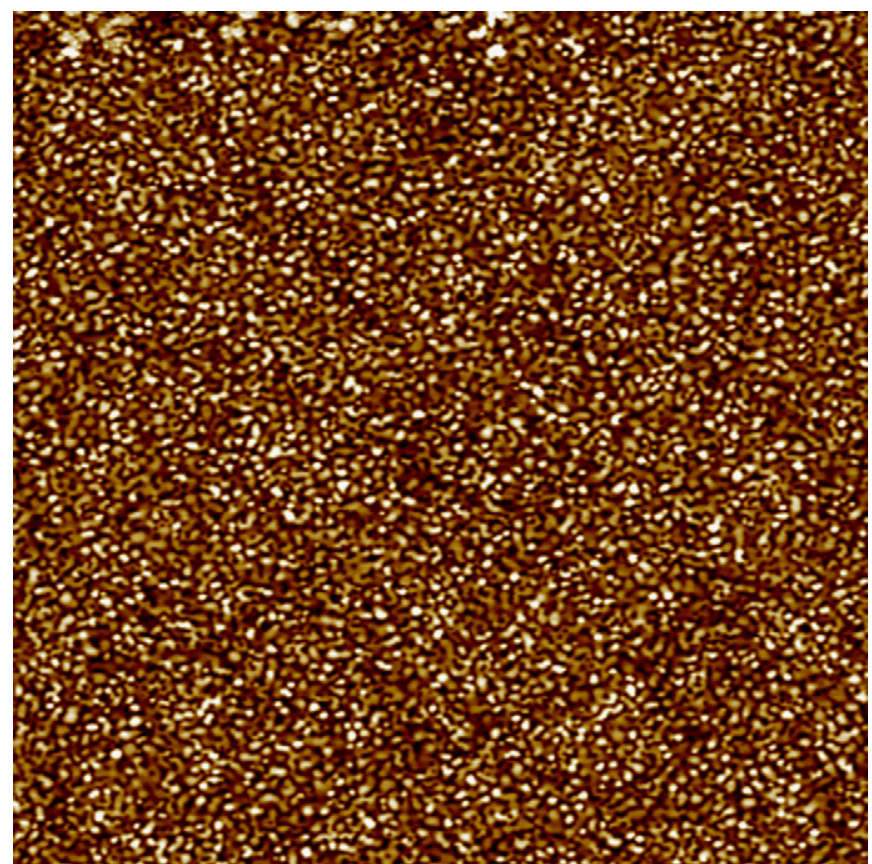

Fig. 1. Surface morphology of the AlN wetting layer. The scan size is $5 \mu \mathrm{m} \times 5 \mu \mathrm{m}$; $30 \mathrm{~nm}$ in $z$-scale.

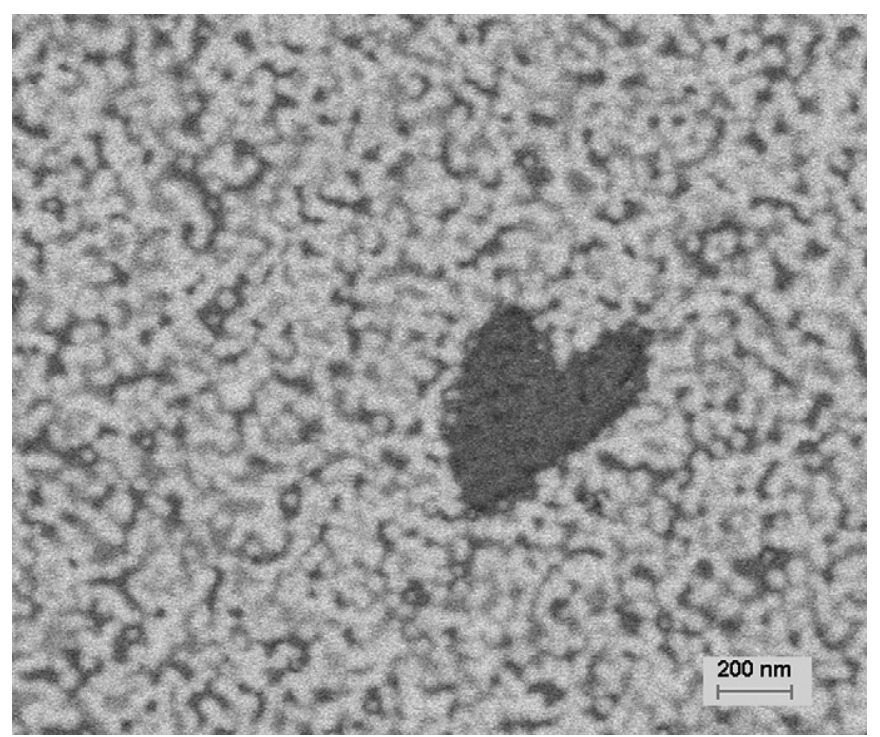

Fig. 2. Contrast imaging (SEM with EsB detector) of a 1 min GaN deposition at 50 mbar. Dark fields represent AlN, whereas the white ones denote GaN.

low-loss BSE with the smallest energy loss resulting from scattering. This enables fine contrast imaging of the smallest compositional differences in materials, hence allowing differentiation between GaN and the underlying AIN layer. The SEM images of the samples grown for $1 \mathrm{~min}$ at $50 \mathrm{mbar}$ are presented in Fig. 2. Areas devoid of GaN show up as dark fields. It was observed that the degree of cladding of the AlN layer by the GaN seeds is different and depends on the reactor pressure. For the lowest pressure investigated, cladding is relatively low as opposed to the sample grown at 125 mbar where the AlN surface is almost completely covered with GaN seeds. In the case of a sample grown at 200 mbar the image is almost identical to that for 125 mbar. 
a

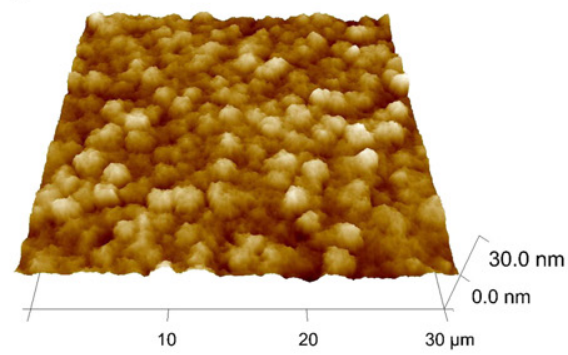

d



g

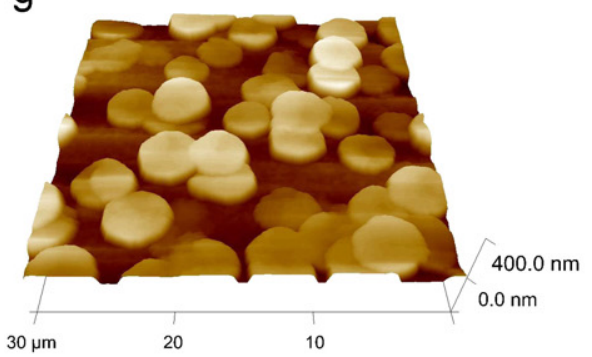

b

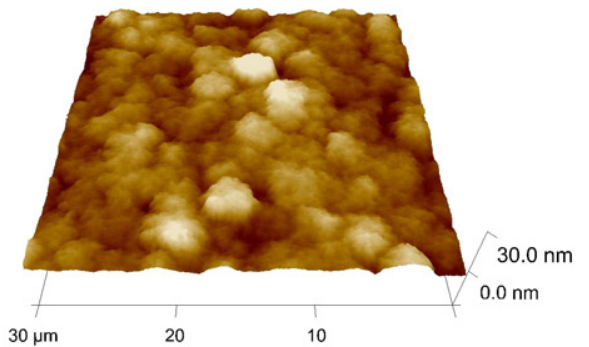

e



h



C



f



i

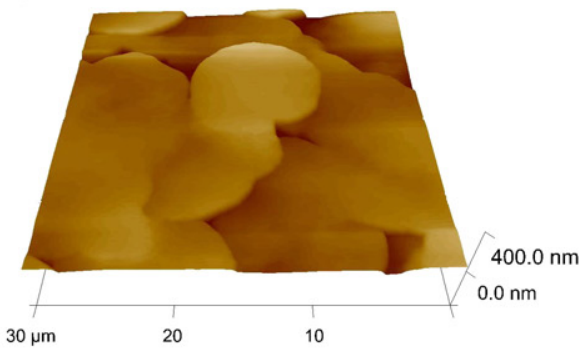

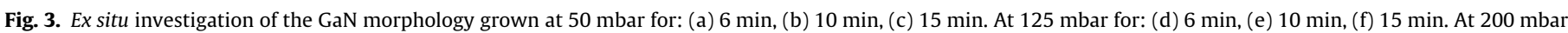

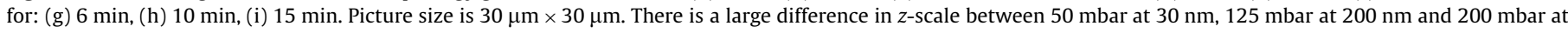
$400 \mathrm{~nm}$.

Considering the growth mechanism at 50 mbar for times of deposition greater than $1 \mathrm{~min}$, it was shown that after $6 \mathrm{~min}$ growth the GaN grains cover the whole surface of the wetting layer. The three-dimensional (3D) growth mode of the deposited GaN can still be observed (Fig. 3a). After 10 min growth at the same pressure the surface roughness still evolves, which means that coalescence is still going on (Fig. 3b). After $15 \mathrm{~min}$ of deposition, the coalescence process was finished and twodimensional (2D) growth mode started, resulting in improvement of the surface roughness (Fig. 3c).

When it comes to epitaxy at 125 mbar, it was shown that after 6 min of deposition the surface is almost completely covered with islands $3.3-3.6 \mu \mathrm{m}$ in diameter and $70-80 \mathrm{~nm}$ in width. At this point the process of island coalescence begins. 3D growth continues also for $10 \mathrm{~min}$ of deposition; in this case the islands' diameter reaches up to 6-7.5 $\mu \mathrm{m}$. Due to the fact that almost the whole surface is covered by GaN blocks, the determination of their height above the AlN surface becomes impossible. In Fig. 3f, which presents the GaN surface after 15 min of deposition, it is seen that the $3 \mathrm{D}$ growth mode is still present. In this case the minimal island diameter was estimated to be $10 \mu \mathrm{m}$.

Similar growth evolution as for the 125 mbar can be observed for the deposition at 200 mbar. Nevertheless, there is a tremendous difference in the rate of the process of coalescence, which takes more time in comparison with growth at 125 mbar. After $6 \mathrm{~min}$ of deposition islands are $4.5-5 \mu \mathrm{m}$ in diameter and $80-120 \mathrm{~nm}$ in height, and a lot of empty spaces between the islands are observed. After 10 min the $3 \mathrm{D}$ growth mode was still being dominant; however, the islands have a diameter of 6.5-7.5 $\mu \mathrm{m}$, which is greater than that observed for $6 \mathrm{~min}$. Unlike in the instance of growth at $125 \mathrm{mbar}$, it is still possible to determine the islands' height, which in this case reaches $230-280 \mathrm{~nm}$. After $15 \mathrm{~min}$ of deposition the islands are grouped together and the differences in their heights and sizes can still be observed, which means that 3D growth mode is still present. Minimal island diameter was estimated to be $11.5 \mu \mathrm{m}$.

But it was not until $28 \mathrm{~min}$ of deposition that the change from 3D to 2D growth mode was observed for both 125 and 200 mbar. The switch point at 125 mbar can be expected earlier than that for 200 mbar but this suggestion requires experimental verification.

The sample deposited at 50 mbar has a coalescence thickness of approximately $0.5 \mu \mathrm{m}$, whereas for samples deposited at 125 and $200 \mathrm{mbar}$ it is $1 \mu \mathrm{m}$. It was also confirmed that for the pressure of 250 mbar the coalescence thickness was estimated to be much higher than $1.7 \mu \mathrm{m}$ (Fig. 4).

Measurement of the surface roughness has been performed by means of the AFM method. The measured quantity is roughness average (RA). The result of the measurement depends on the location and size of the scanned area. The measurements have been performed at a randomly selected area of size $30 \mu \mathrm{m} \times 30$ $\mu \mathrm{m}$, located somewhere in the middle of the sample. This particular size was chosen because of its similarity to the dimensions of typical devices built using GaN-based structures. An additional RA measurement has been performed on the area of size $5 \mu \mathrm{m} \times 5 \mu \mathrm{m}$ selected within the previously measured one. The reason for this is the fact that the diameter of this area is 


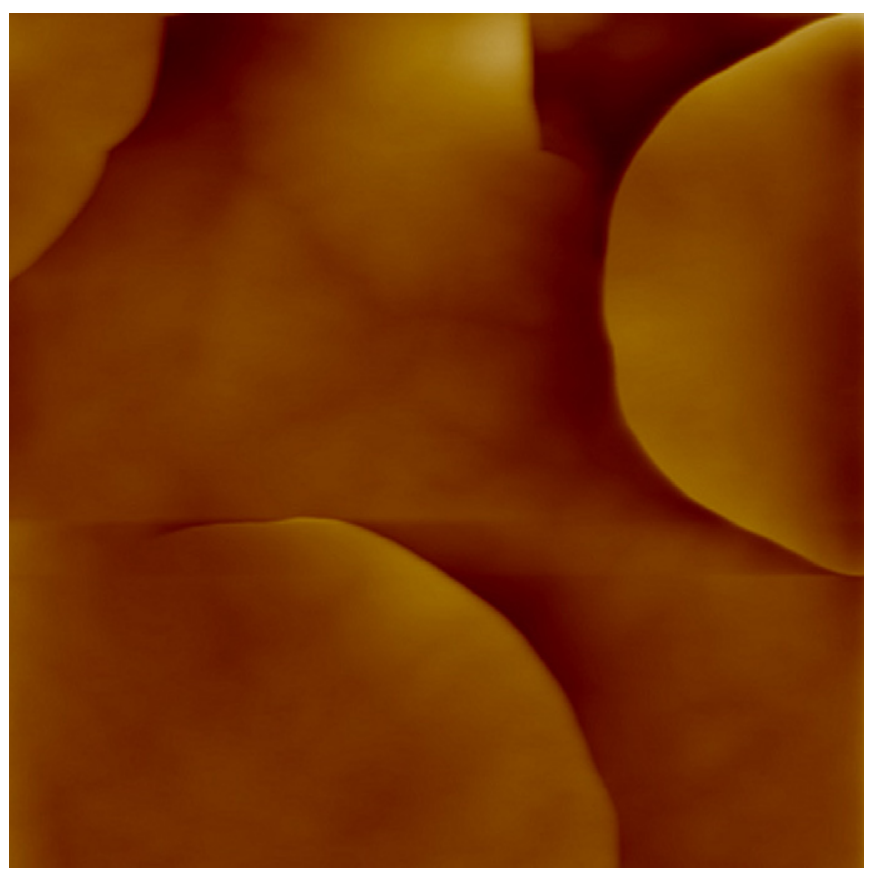

Fig. 4. Surface morphology of the $1.7 \mu \mathrm{m}$ thick GaN deposition at 250 mbar. The scan size is $90 \mu \mathrm{m} \times 90 \mu \mathrm{m} ; 200 \mathrm{~nm}$ in $z$-scale.

Table 1

The RA values measured for samples deposited at different reactor pressures.

\begin{tabular}{lll}
\hline $\begin{array}{l}\text { Pressure } \\
\text { (mbar) }\end{array}$ & Scanned area & \\
\cline { 2 - 3 } & $\begin{array}{l}30 \mu \mathrm{m} \times 30 \mu \mathrm{m} \\
(\mathrm{nm})\end{array}$ & $\begin{array}{l}5 \mu \mathrm{m} \times 5 \mu \mathrm{m} \\
(\mathrm{nm})\end{array}$ \\
\hline 50 & 1.7 & 0.48 \\
125 & 1.49 & 0.30 \\
200 & 1.5 & 0.28 \\
\hline
\end{tabular}

similar to the typical distances between the ohmic contacts in HEMT devices. The results achieved for $1.7 \mu \mathrm{m}$ thick GaN layers depend only on the deposition pressure and the size of the scanned area and are presented in Table 1.

The observed decrease in RA values for both selected areas, which occurs while going from 50 to $125 \mathrm{mbar}$, may be interpreted as the improvement of the state of the surface. The noticeable difference in RA values for all pressures measured for both areas may result from the fact that the linear dimension of the $5 \mu \mathrm{m} \times 5 \mu \mathrm{m}$ area is less than the diameter of a single island. In Fig. 5(a,b) the surface relief of the $5 \mu \mathrm{m} \times 5 \mu \mathrm{m}$ area, where RA measurements have been performed, for samples deposited at 50 and $200 \mathrm{mbar}$, is presented. For the sample deposited at $50 \mathrm{mbar}$ a greater density of atomic steps is visible in comparison to the sample deposited at 200 mbar. Results presented in Table 1 and Fig. 5a and b may suggest that there exists a correlation on the one hand between the island diameter and the number of atomic steps on the surface and on the other hand the surface roughness.

Defect-selective etching (DSE) of the GaN surface in the molten $\mathrm{NaOH}-\mathrm{KOH}$ eutectic with addition of $10 \%$ of $\mathrm{MgO}$ powder was used in order to reveal dislocations on the epilayers surface [7]. The AFM images of the etched epilayer surfaces allowed estimation of the dislocation density. The total dislocation density (TDD), of GaN on N-type substrates, estimated by the DSE method, was found to be almost identical $\left(\sim 3 \times 10^{9} \mathrm{~cm}^{-2}\right)$ for all investigated samples. It was discovered that in all samples, there
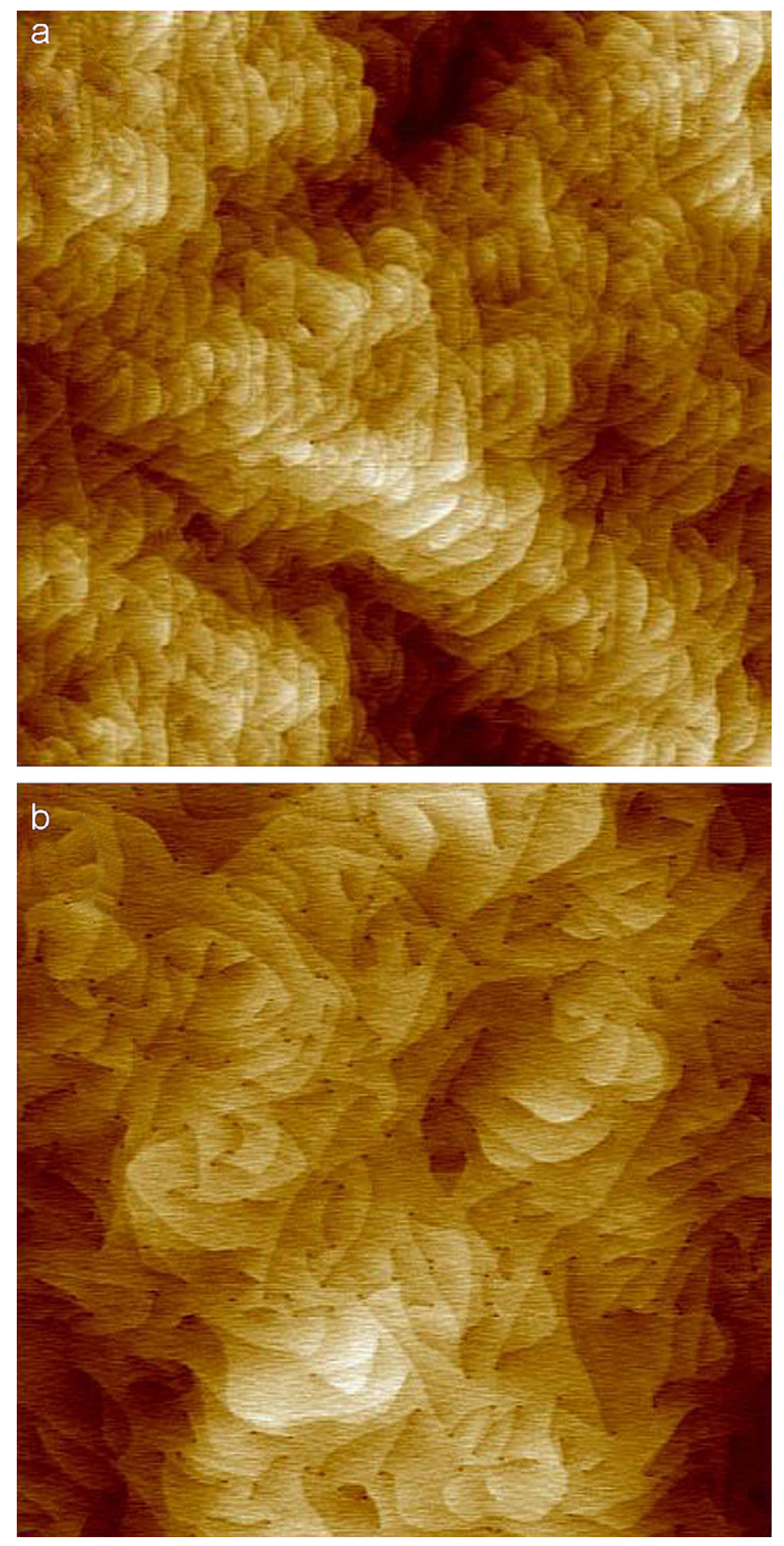

Fig. 5. Surface morphology of GaN grown at (a) 50 mbar and (b) 200 mbar. The scan size is $5 \mu \mathrm{m} \times 5 \mu \mathrm{m} ; 10 \mathrm{~nm}$ in $z$-scale.

\section{Table 2}

XRD FWHM results (in arcsec) for GaN layers deposited on $4 \mathrm{H}-\mathrm{SiC}$ at 50, 125 and 200 mbar.

\begin{tabular}{lcc}
\hline$p_{\mathrm{GaN}}(\mathrm{mbar})$ & $\sigma_{\perp}(\operatorname{arsec})$ & $\sigma_{\|}$(arsec) \\
\hline 50 & 119 & 148 \\
125 & 87 & 157 \\
200 & 46 & 156 \\
\hline
\end{tabular}

were almost twice as many screw and mixed type dislocations as edge type ones. For the 200 mbar growth on semi-insulating substrate, TDD was calculated to be $2.1 \times 10^{9} \mathrm{~cm}^{-2}$, with three fold fewer edge type dislocations than in the others. 
Table 3

Sheet carrier concentration, mobility, breakdown voltage and RF measurements for the HEMT structures grown at the different pressures.

\begin{tabular}{|c|c|c|c|c|c|}
\hline GaN pressure(mbar) & $n_{\mathrm{s}}\left(\mathrm{cm}^{-3}\right)$ & $\mu_{\mathrm{e}}\left(\mathrm{cm}^{2} / \mathrm{Vs}\right)$ & $\operatorname{Vbr}(V)$ & $\mathrm{Ft}(\mathrm{GHz})$ & Fmax $(\mathrm{GHz})$ \\
\hline 50 & $8 \times 10^{12}$ & 1250 & 55 & 6.9 & 12.9 \\
\hline 125 & $7.3 \times 10^{12}$ & 1500 & 65 & 7.2 & 16.7 \\
\hline 200 & $5.5 \times 10^{12}$ & 860 & 68 & 10.4 & 15.5 \\
\hline
\end{tabular}

The GaN/Al ${ }_{0.23} \mathrm{GaN} / \mathrm{AlN} / \mathrm{GaN}$-based HEMT epilayer structures with a $1.7 \mu \mathrm{m}$ thick GaN buffer (grown at 50, 125 or 200 mbar) were prepared on semi-insulating substrates in exactly the same growth conditions as those previously mentioned.

These structures have been used to perform X-ray measurements consisting of $\omega / 2 \theta$ and $\omega$ scans in the vicinity of the $\left(\begin{array}{lll}0 & 0 & 2\end{array}\right)$ $\mathrm{GaN}$ reflection using a double-crystal diffractometer [8]. These measurements have been performed employing the HRXRD diffractometer equipped with a Ge 440 Bartels monochromator using $\mathrm{CuK}_{\alpha 1}$ radiation (wavelength $=0.15405 \mathrm{~nm}$ ) from a $2.2 \mathrm{~kW}$ ceramic Philips tube.

Results of the X-ray measurements are presented in Table 2. For this method, the broadening of the symmetric reciprocal lattice point (RLP) of the GaN epitaxial film in the direction perpendicular to the surface $\sigma_{\perp}$ depends on the thickness of the coherently scattering regions and a heterogeneous strain measured along the $c$-axis [9]. On the other hand, the broadening of RLP parallel to the surface $\sigma_{\text {II }}$ is influenced by the tilt, i.e. an out of plane misorientation [10].

Using a triple-axis high resolution X-ray diffractometer it is possible to determine not only the broadening $\sigma_{\perp}$ of the 002 RLP by measuring the FWHM of the diffraction peak obtained in the $\omega / 2 \theta$-scan mode, but also the broadening $\sigma_{\text {II }}$ of this RLP by measuring its FWHM obtained in the $\omega$ scan mode.

Results show the influence of GaN growth pressure on its crystallographic structure. Even though the growth pressure does not influence the epilayer mosaicity and does not reduce grain misorientation (twist and tilt), which can be deduced from the analysis of $\sigma_{\text {II }}$ FWHM (Table 2), a strong dependence between GaN growth pressure and its crystal quality in the direction perpendicular to the surface is observed. The best crystal quality is observed for the sample grown at $200 \mathrm{mbar}$ (FWHM $=46$ arcsec).

Sample HEMT devices with $L_{\mathrm{SD}} / L_{\mathrm{SG}} / W_{\mathrm{G}} / L_{\mathrm{G}}=(3 / 1 / 250 / 0.4) \mu \mathrm{m}$ were fabricated from the HEMT epilayer structures. In the HEMTs the barrier layers were intentionally Si-doped to $n=1 \times 10^{18}$ $\mathrm{cm}^{-3}$. Ti/Al/ Ni/Au (150/1500/400/700) $\AA$ were used for the source and drain ohmic contacts and were annealed at $850{ }^{\circ} \mathrm{C}$ for $30 \mathrm{~s}$ in forming gas. Ni/Au (500/2000) $\AA$ Schottky gates were then metallized. A reactive ion-etched mesa was selected for device isolation. The DC current-voltage characteristics of the fabricated HEMTs were measured by means of an HP 4142B semiconductor parameter analyzer, while the small signal radio frequency characteristics were measured by means of an Agilent N5230A network analyzer. The measurements were performed on a Cascade Microtech/Alessi RF1 microwave probe station with Cascade Microtech GSG150 probes. Both sheet carrier concentration $\left(n_{\mathrm{s}}\right)$ and mobility $\left(\mu_{\mathrm{e}}\right)$ values were measured by the standard Van der Pauw-Hall technique at room temperature. Most important results of the measurement are presented in Table 3.

In order to verify the influence of the reactor pressure, at which the GaN buffer layer has been deposited on the resistance of the HEMT structure, a series of breakdown voltage measurements have been performed. It was found that there exists a dependence between deposition pressure and measured $\mathrm{Vbr}$ value (Table 3 ). The highest breakdown voltage $\mathrm{Vbr}$ has been measured for the device prepared on a 200 mbar GaN buffer. Such

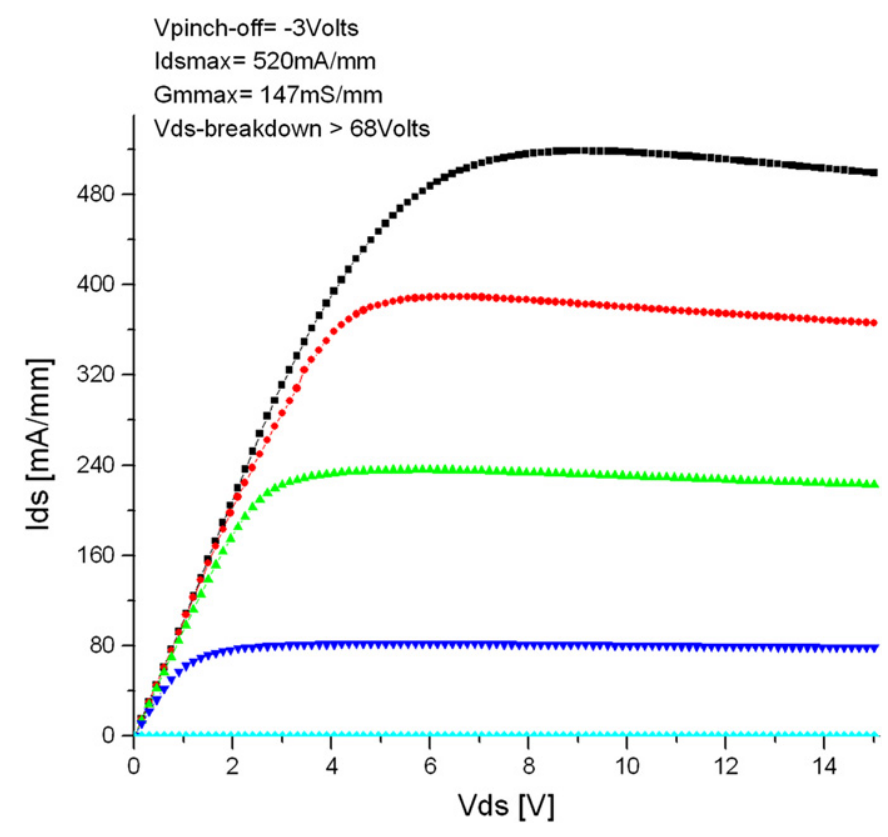

Fig. 6. Drain characteristics of HEMT with GaN buffer grown at 200 mbar.

a high breakdown voltage value is attributed to the quality of the GaN buffer layer (Fig. 6).

\section{Summary}

GaN layers on a SiC substrate grown in various reactor pressure conditions have been investigated. It was found that GaN layer coalescence thickness depends on this pressure. Growth at higher pressures requires a thicker GaN layer to observe a switch from 3D to 2D GaN growth mode. This switch point serves as an indicator for the end of the coalescence process. It was also proved that the increase in the growth pressure from 50 to 125 mbar improves the surface roughness, whereas above this value no noticeable improvement is observed. From the presented results of AFM measurements one can draw conclusions that for reactor pressures above 125 mbar the surface quality does not change, whereas X-ray measurements still show further improvement of crystal quality of the investigated GaN layer as the pressure increases. All these collected results along with the ones derived from the device characterization, especially breakdown voltage, suggest that the growth at 200 mbar is the most suitable for HEMTs with the $1.7 \mu \mathrm{m}$ thick GaN buffer layer.

\section{References}

[1] K Hiramatsu, S Itoh, H Amano, I Akasaki, N Kuwano, T Shiraishi, K Oki, J. Cryst. Growth 115 (1991) 628

[2] S-W. Kim, T. Yamada, K. Haga, M. Akatsu, T. Suzuki, Proc. Int, Workshop on nitride. semiconductors, in: Proceedings of the IPAP Conference Series 1, 2000, pp. 247-250.

[3] Y. Suk Cho, H. Hardtdegen, N. Kaluza, R. Steins, G. Heidelberger, H. Luth J. Cryst. Growth 307 (2007) 6-13. 
[4] A.E. Wickenden, D.D. Koleske, R.L. Henry, R.J. Gorman, J.C. Culbertson, M.E. Twigg, J. Electron. Mater. 28 (3) (1999).

[5] S. Boeykens, M.R. Leys, M. Germain, R. Belmans, G Borghs, J. Cryst. Growth 272 (2004) 312-317.

[6] B. Moran, F. Wu, A.E. Romanov, U.K. Mishra, S.P. Denbaars, J.S. Speck, J. Cryst. Growth 273 (2004) 38-47.
[7] G. Kamler, J.L. Weyher, I. Grzegory, E. Jezierska, T. Wosinski, J. Cryst. Growth 245 (2002) 21-24.

[8] T. Matsushita, S. Kikuta, K. Kohra, J. Phys. Soc. Japan 30 (1971) 1136.

[9] T Metzger, et al., Phil. Mag. A 77 (4) (1998) 1013-1025.

[10] T.H. De Keijser, et al., J. Appl. Crystallogr. 16 (1983) 309. 